\title{
A non-standard FETI-DP mortar method for Stokes problem
}

\author{
E. CHACÓN VERA* ${ }^{*}$ and D. FRANCO CORONIL ${ }^{\dagger}$
}

Received April 25, 2012

\begin{abstract}
The trace spaces $H^{1 / 2}$ and $H_{00}^{1 / 2}$ play a key role in the FETI and mortar families of domain decomposition methods. However, a direct numerical evaluation of these norms is usually avoided. On the other hand, and for stability issues, the subspace of functions for which their jumps across the interfaces of neighbouring subdomains belong to these trace spaces yields a more suitable framework than the standard broken Sobolev space. Finally, the nullity of these jumps is usually imposed via Lagrange multipliers and using the pairing of the trace spaces with their duals. A direct computation of these pairings can be performed using the Riesz-canonical isometry. In this work we consider all these ingredients and introduce a domain decomposition method that falls into the FETI-DP mortar family. The application is to the incompressible Stokes problem and we see that continuous bounds are replicated at the discrete level. As a consequence, no stabilization is required. Some numerical tests are finally presented.
\end{abstract}

Keywords: FETI-DP methods, mortar methods, trace norms, freefem++

\section{Introduction}

Given a computational domain $\Omega\left(\Omega \subset \mathbb{R}^{2}\right.$ bounded polygonal to ease presentation) we denote by $H_{0}^{1}(\Omega)$ the closure in the standard Sobolev space $H^{1}(\Omega)$ of all the smooth functions with support inside $\Omega$. As usual, we split $\Omega$ into open polygonal subdomains $\Omega=\bigcup_{s=1}^{S} \Omega^{s}$ with $\Omega^{s} \cap \Omega^{t}=\varnothing(s \neq t)$, denote by $\partial \Omega^{s}$ the boundary of any $\Omega^{s}$ and let $\Gamma_{s, t}=\partial \Omega^{s} \cap \partial \Omega^{t}$ be either an edge (i.e., a segment), a crosspoint or empty. Finally, consider $\mathscr{E}_{0}=\left\{\Gamma_{e}\right\}_{e=1, \ldots . E}$ the sorted set of all edges inside $\Omega$, also known as the skeleton of the decomposition. We

\footnotetext{
*Dpto. Matemáticas, Facultad de Matemáticas, Universidad de Murcia, Campus Espinardo, 30100 Murcia, SPAIN. Email: eliseo@um.es

†Dpto. Ecuaciones Diferenciales y Análisis Numérico, Facultad de Matemáticas, Universidad de Sevilla, Tarfia sn.41012 Sevilla, SPAIN. Email: franco@us.es

Research partially funded by Spanish government MEC Research Project MTM200907719.
} 


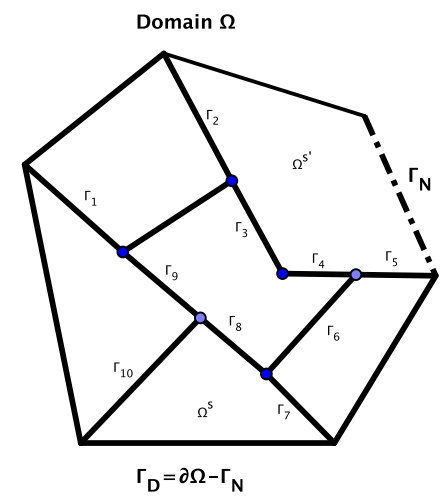

Figure 1. Example of domain decomposition where cross points are marked with big dots and the skeleton is the set of all the $\Gamma_{i}$ for $i=1,2, \ldots, 10$. In the case of Dirichlet Boundary conditions we have $\Gamma_{N}=\varnothing$.

assume that each $\Omega^{s}$ is of area $\mathscr{O}\left(H^{2}\right)$ and shape regular while each $\Gamma_{e}$ is of length $\mathscr{O}(H)$ for some fixed $H>0$ (see Fig. 1 for example).

Let us denote by $[v]_{\Gamma_{e}}$ the jump across $\Gamma_{e}$ and consider the Hilbert space

$X=\left\{v \in L^{2}(\Omega): v^{s}=v_{\left.\right|_{\Omega^{s}}} \in H^{1}\left(\Omega^{s}\right) \cap H_{0}^{1}(\Omega) \forall s,[v]_{\Gamma_{e}} \in H_{00}^{1 / 2}\left(\Gamma_{e}\right) \forall \Gamma_{e} \in \mathscr{E}_{0}\right\}$

with the graph norm

$$
\|v\|_{X}=\left\{\sum_{s=1}^{S}\left\|v^{s}\right\|_{1, \Omega^{s}}^{2}+\sum_{e=1}^{E}\left\|[v]_{\Gamma_{e}}\right\|_{1 / 2,00, \Gamma_{e}}^{2}\right\}^{1 / 2} .
$$

Here $\|\cdot\|_{1 / 2,00, \Gamma_{e}}$ is the norm induced by the scalar product $(\cdot, \cdot)_{1 / 2,00, \Gamma_{e}}$ on $H_{00}^{1 / 2}\left(\Gamma_{e}\right)$ :

$$
(w, v)_{1 / 2,00, \Gamma_{e}}=(w, v)_{1 / 2, \Gamma_{e}}+\int_{\Gamma_{e}} \frac{w(x) v(x)}{d\left(x, \partial \Gamma_{e}\right)} \mathrm{d} x
$$

where $d\left(x, \partial \Gamma_{e}\right)$ is the distance of $x$ to the boundary of $\Gamma_{e}$ and

$$
(w, v)_{1 / 2, \Gamma_{e}}=\int_{\Gamma_{e}} w(x) v(x) \mathrm{d} x+\int_{\Gamma_{e}} \int_{\Gamma_{e}} \frac{(w(x)-w(y))(v(x)-v(y))}{|x-y|^{2}} \mathrm{~d} x \mathrm{~d} y .
$$


To simplify notation, we write $\{w, v\}_{\Gamma_{e}}=(w, v)_{1 / 2,00, \Gamma_{e}}$. Poincare's inequality and the control we have on the jumps, interconnecting all subdomains, allow the use on $X$ of the equivalent norm $|v|_{X}^{2}=(v, v)_{X}$, where $(\cdot, \cdot)_{X}$ is the scalar product given by

$$
(u, v)_{X}=\sum_{s=1}^{S}\left(\nabla u^{s}, \nabla v^{s}\right)_{\Omega^{s}}+\sum_{e=1}^{E}\left\{[u]_{\Gamma_{e}},[v]_{\Gamma_{e}}\right\}_{\Gamma_{e}} \quad \forall u, v \in X .
$$

Bernardi et al. in [3] and [7] used the space $X$ and the (Riesz) identification of the Hilbert space $H_{00}^{-1 / 2}\left(\Gamma_{e}\right)$ with its dual $H_{00}^{1 / 2}\left(\Gamma_{e}\right)$ in order to present a continuous framework for a domain decomposition method for elliptic equations. Their method falls into the FETI-DP mortar family: FETI-DP alike because only continuity at cross points is imposed and mortar alike because nonmatching meshes at interfaces are allowed, although a different scalar product for the mortaring process is used. In this approach, the continuous analysis holds for internal approximations even in the case of non conforming meshes and makes theory remarkably simple. Another interesting property is that the stability of the discretization is independent of the varying mesh sizes, i.e., it does not matter which side is the mortar. On the other hand, and only in the presence of crosspoints, we recover the standard mesh dependency bounds in terms of $\log (H / h)$ that appear in FETI-DP methods (see $[9,15])$. A similar approach was introduced by Braess et al. in [5]; although they used a mesh dependent $L^{2}\left(\Gamma_{e}\right)$ norm to approximate the $H_{00}^{1 / 2}\left(\Gamma_{e}\right)$ norm. See also the works by Farhat et al. [9] and Dryja and Widlund [8] for the origin of the FETI-DP mortar method.

In this paper we extend the ideas in [3] and [7] to the case of the incompressible Stokes equations showing that the same properties hold. Our analysis will be presented in the two dimensional setting to simplify the presentation. These main ideas extend easily to the three dimensional situation although a detailed study is required. Finally some numerical tests are shown as a conclusion.

\section{Incompressible Stokes equations}

The zero average of the pressure imposes a global condition on the pressure space and, as a consequence, the splitting of the computational subdomain for the pressure is more difficult. We introduce a new variable in the Stokes equations that sets free the pressure space from this restriction.

Incompressible Stokes equations with homogeneous boundary conditions 
amount to find $u \in \mathbf{H}_{0}^{1}(\Omega)=\left(H_{0}^{1}(\Omega)\right)^{2}$ and $p \in L^{2}(\Omega)$ such that

$$
\begin{aligned}
(\nabla u, \nabla v)_{\Omega}-(p, \operatorname{div}(v))_{\Omega} & =(f, v)_{\Omega} & & \forall v \in \mathbf{H}_{0}^{1}(\Omega) \\
-(q, \operatorname{div}(u))_{\Omega} & =0 & & \forall q \in L^{2}(\Omega) \\
\int_{\Omega} p & =0 . & &
\end{aligned}
$$

We better accommodate the restriction on the pressure by adding a new scalar unknown as follows: we look for a pair of values $(u, \tau) \in \mathbf{H}_{0}^{1}(\Omega) \times \mathbb{R}$ and $p \in L^{2}(\Omega)$ such that

$$
\begin{array}{ccrl}
(\nabla u, \nabla v)_{\Omega}-(p, \operatorname{div}(v))_{\Omega}+t\left(\tau-\int_{\Omega} p\right) & =(f, v)_{\Omega} & & \forall(v, t) \in \mathbf{H}_{0}^{1}(\Omega) \times \mathbb{R} \\
-(q, \operatorname{div}(u))_{\Omega}-\tau \int_{\Omega} q=0 & & \forall q \in L^{2}(\Omega) .
\end{array}
$$

A saddle point problem emerges with the incorporation of this new variable $\tau$ when the first equation is written as

$$
(\nabla u, \nabla v)_{\Omega}+\tau t-(p, \operatorname{div}(v))_{\Omega}-t \int_{\Omega} p=(f, v)_{\Omega}
$$

Set $W=\mathbf{H}_{0}^{1}(\Omega) \times \mathbb{R}$ and let $\underline{v}=(v, t) \in W$ any element of $W$. We norm $W$ by using

$$
\|\underline{v}\|_{W}^{2}=\|(v, t)\|_{W}^{2}=\|\nabla v\|_{0, \Omega}^{2}+t^{2}
$$

and let $(\cdot, \cdot)_{W}: W \times W \mapsto \mathbb{R}$ be the scalar product on $W$, i.e.,

$$
(\underline{u}, \underline{v})_{W}=((u, \tau),(v, t))_{W}=(\nabla u, \nabla v)_{\Omega}+\tau t .
$$

Finally, we take our restriction as $b: W \times L^{2}(\Omega) \mapsto \mathbb{R}$ given by

$$
b(q, \underline{v})=b(q,(v, t))=-(q, \operatorname{div}(v))_{\Omega}-t \int_{\Omega} q .
$$

Then, we look for $\underline{u}=(u, \tau) \in W$ and $p \in L^{2}(\Omega)$ such that for all $\underline{v}=(v, t) \in W$ and $q \in L^{2}(\Omega)$

$$
\begin{aligned}
(\underline{u}, \underline{v})_{W}+b(p, \underline{v}) & =(f, v)_{\Omega} \\
b(q, \underline{u}) & =0 .
\end{aligned}
$$


Lemma 2.1. There exists a positive constant $\beta>0$ such that for all $p \in$ $L^{2}(\Omega)$

$$
\sup _{(v, t) \in W} \frac{b(p,(v, t))}{\|(v, t)\|_{W}} \geqslant \sup _{v \in \mathbf{H}_{0}^{1}(\Omega), t \in \mathbb{R}} \frac{b(p,(v, t))}{\left(\|\nabla v\|_{0, \Omega}^{2}+t^{2}\right)^{1 / 2}} \geqslant \beta\|p\|_{0, \Omega} .
$$

As a consequence, the saddle point problem (2.1)-(2.2) is well posed and its unique solution is the one for the original Stokes problem with Dirichlet homogeneous boundary conditions.

Proof. Take $p_{\Omega}=|\Omega|^{-1} \int_{\Omega} p \mathrm{~d} x$, then $p-p_{\Omega} \in L_{0}^{2}(\Omega)$ and there exists $v^{*} \in$ $\mathbf{H}_{0}^{1}(\Omega)$ that gives the inf-sup condition for $p-p_{\Omega}$; take also $t^{*}=-p_{\Omega}$, then the result follows in a standard way.

\subsection{Multidomain formulation to the Stokes problem}

Let us define the spaces for our formulation. For the elliptic part set $\mathbf{V}=\mathbf{X} \times \mathbb{R}$, where $\mathbf{X}=X \times X$, and represent by $\underline{v}=(v, t)$ any element of $\mathbf{V}$ where $v \in \mathbf{X}$ and $t \in \mathbb{R}$. Obviously, $\mathbf{V}$ is Hilbert space with norm $\|\underline{v}\|_{\mathbf{V}}^{2}=|v|_{\mathbf{X}}^{2}+t^{2}$. For the pressure variables we consider $\mathbf{M}=\prod_{s=1}^{S} L^{2}\left(\Omega^{s}\right)\left(\approx L^{2}(\Omega)\right)$. For each $\Gamma_{e} \in \mathscr{E}_{0}$ we take $\mathbf{H}_{00}^{1 / 2}\left(\Gamma_{e}\right)=\left(H_{00}^{1 / 2}\left(\Gamma_{e}\right)\right)^{2}$ and handle the Lagrange multipliers for the jumps in $\mathbf{N}=\prod_{e=1}^{E} \mathbf{H}_{00}^{1 / 2}\left(\Gamma_{e}\right)$ with scalar product

$$
(\lambda, \mu)_{\mathbf{N}}=\sum_{e=1}^{E}\left\{\lambda_{e}, \mu_{e}\right\}_{\Gamma_{e}} \quad \forall \lambda, \mu \in \mathbf{N}
$$

In our new multidomain formulation at the continuous level we add, only in the presence of crosspoints, the jumps to the elliptic terms and replace the pairings $H_{00}^{-1 / 2}(\Gamma)-H_{00}^{1 / 2}(\Gamma)$ for the normal fluxes on the edges by the scalar product in $H_{00}^{-1 / 2}(\Gamma)$. As a consequence, all terms are suitable to compute in a Galerkin approach and without changing the regularity on the edge terms. 
We look for $\underline{u}=(u, \tau) \in \mathbf{V}, p=\left\{p^{s}\right\}_{s} \in \mathbf{M}$ and $\lambda=\left\{\lambda_{e}\right\}_{e} \in \mathbf{N}$ such that

$$
\begin{aligned}
& \sum_{s=1}^{S}\left(\nabla u^{s}, \nabla v^{s}\right)_{\Omega^{s}}+\sum_{e=1}^{E}\left\{[u]_{\Gamma_{e}},[v]_{\Gamma_{e}}\right\} \Gamma_{\Gamma_{e}}+\tau t \\
&-\sum_{s=1}^{S}\left(p^{s}, \operatorname{div}\left(v^{s}\right)\right)_{\Omega^{s}}-t \sum_{s=1}^{S} \int_{\Omega^{s}} p^{s}+\sum_{e=1}^{E}\left\{\lambda_{e},[v]_{\Gamma_{e}}\right\}_{\Gamma_{e}}=\sum_{s=1}^{S}\left(f, v^{s}\right)_{\Omega^{s}} \\
&-\sum_{s=1}^{S}\left(q^{s}, \operatorname{div}\left(u^{s}\right)\right)_{\Omega^{s}}-\tau \sum_{s=1}^{S} \int_{\Omega^{s}} q^{s}=0 \\
& \sum_{e=1}^{E}\left\{\mu_{e},[u]_{\Gamma_{e}}\right\}_{\Gamma_{e}}=0
\end{aligned}
$$

for all $\underline{v}=(v, t) \in \mathbf{V}, q=\left\{q^{s}\right\}_{s} \in \mathbf{M}$ and $\mu=\left\{\mu_{e}\right\}_{e} \in \mathbf{N}$. In a more compact form, taking $F(\underline{v})=\sum_{s=1}^{S}\left(f, v^{s}\right)_{\Omega^{s}}$, the problem is

$$
\left\{\begin{aligned}
\text { Find }(\underline{u}, p, \lambda) \in \mathbf{V} \times \mathbf{M} \times \mathbf{N} \text { such that } \\
(\underline{u}, \underline{v}) \mathbf{V}+b(p, \underline{v})+c(\lambda, \underline{v})=F(\underline{v}) \\
b(q, \underline{u})=0 \\
c(\mu, \underline{u})=0 \\
\text { for all }(\underline{v}, q, \mu) \in \mathbf{V} \times \mathbf{M} \times \mathbf{N} .
\end{aligned}\right.
$$

where

$$
\begin{aligned}
& (\underline{u}, \underline{v}) \mathbf{v}=\sum_{s=1}^{S}\left(\nabla u^{s}, \nabla v^{s}\right)_{\Omega^{s}}+\sum_{e=1}^{E}\left\{[u]_{\Gamma_{e}},[v]_{\Gamma_{e}}\right\}_{\Gamma_{e}}+\tau t \\
& b(p, \underline{v})=-\sum_{s=1}^{S}\left(p^{s}, \operatorname{div}\left(v^{s}\right)\right)_{\Omega^{s}}-t \sum_{s=1}^{S} \int_{\Omega^{s}} p^{s} \\
& c(\mu, \underline{u})=c(\mu, u)=\sum_{e=1}^{E}\left\{\mu_{e},[u]_{\Gamma_{e}}\right\}_{\Gamma_{e}} .
\end{aligned}
$$

Inf-sup conditions for $b$ and $c$ are easily checked. Moreover, the inf-sup for the bilinear form $b$ is reached with a function $v \in \mathbf{V}$ with zero jumps while the inf-sup for bilinear form $c$ is reached with a function $v \in \mathbf{V}$ with nonzero jumps, see Lemma 2.9 of [2] for instance. These facts give uniqueness of solution and make the formulation equivalent to that of the incompressible Stokes problem on the global domain $\Omega$.

Next, if we call dual variables the Lagrange multipliers and the rest primal variables we eliminate the primal variables in terms of the dual variables. 
That is to say, we obtain a dual problem that once solved will give the correct boundary data for the primal variables. Using standard operator notation, (2.5) is

$$
\left\{\begin{array}{rlrl}
\text { Find }(\underline{u}, p, \lambda) \in \mathbf{V} \times \mathbf{M} \times \mathbf{N} \text { such that } \\
A \underline{u}+B^{*} p+C^{*} \lambda=F & & \text { in } \mathbf{V}^{*} \\
B \underline{u} & =0 & & \text { in } \mathbf{M}^{*} \\
C \underline{u} & =0 & & \text { in } \mathbf{N}
\end{array}\right.
$$

and the dual problem for $\lambda$ is an equation on $\mathbf{N}$ in the form $S \lambda=\ell$, where $S$ and $\ell$ are given by

$$
\begin{aligned}
& S=\left(C A^{-1} C^{*}\right)-\left(C A^{-1} B^{*}\right)\left(B A^{-1} B^{*}\right)^{-1}\left(B A^{-1} C^{*}\right) \\
& \ell=\left(C A^{-1} F\right)-\left(C A^{-1} B^{*}\right)\left(B A^{-1} B^{*}\right)^{-1}\left(B A^{-1} F\right) .
\end{aligned}
$$

Theorem 2.1. Let be $S: \mathbf{N} \rightarrow \mathbf{N}$ defined as above. Then $S$ is a self-adjoint positive definite operator and there exists a constant $\sigma>0$ that only depends on the inf-sup conditions for bilinear forms $b$ and $c$ such that

$$
\sigma^{2}\|\lambda\|_{\mathbf{N}}^{2} \leqslant(S \lambda, \lambda)_{\mathbf{N}} \leqslant\|\lambda\|_{\mathbf{N}}^{2} \quad \forall \lambda \in \mathbf{N}, \quad \lambda \neq 0 .
$$

As a consequence, our dual problem is well posed and has a unique solution that gives the correct Lagrange multipliers for (2.5).

Proof. By the construction of $S$ it is clear that it is self-adjoint.

Let us prove that $S$ is positive definite. For any $\lambda \in \mathbf{N}, \lambda \neq 0$ we have

$$
\begin{aligned}
S \lambda & =\left(C A^{-1} C^{*}\right) \lambda-\left(C A^{-1} B^{*}\right)\left(B A^{-1} B^{*}\right)^{-1}\left(B A^{-1} C^{*}\right) \lambda \\
& =\left(C A^{-1} C^{*}\right) \lambda+\left(C A^{-1} B^{*}\right) p_{\lambda}=-C \underline{u} \lambda
\end{aligned}
$$

where

$$
p_{\lambda}=-\left(B A^{-1} B^{*}\right)^{-1}\left(B A^{-1} C^{*}\right) \lambda, \quad \underline{u}_{\lambda}=-A^{-1} C^{*} \lambda-A^{-1} B^{*} p_{\lambda} .
$$

As a consequence, $\underline{u}_{\lambda} \in \mathbf{V}$ and $p_{\lambda} \in \mathbf{M}$ satisfy

$$
\begin{aligned}
A \underline{u}_{\lambda}+B^{*} p_{\lambda}+C^{*} \lambda & =0 & & \text { in } \mathbf{V}^{*} \\
B \underline{u}_{\lambda} & =0 & & \text { in } \mathbf{M}^{*} \\
C \underline{u}_{\lambda} & =-S \lambda & & \text { in } \mathbf{N} .
\end{aligned}
$$

Thanks to the uniqueness of solution, $S \lambda \neq 0$, because $S \lambda=0$ would imply $\underline{u}_{\lambda}=0, p_{\lambda}=0$ and $\lambda=0$ while we assume $\lambda \neq 0$. Then,

$$
\begin{aligned}
(S \lambda, \lambda)_{\mathbf{N}}=\left(-C \underline{u}_{\lambda}, \lambda\right)_{\mathbf{N}} & =-\left\langle C^{*} \lambda, \underline{u}_{\lambda}\right\rangle_{\mathbf{V}} \\
& =\left\langle A \underline{u}_{\lambda}+B^{*} p_{\lambda}, \underline{u}_{\lambda}\right\rangle_{\mathbf{V}}=\left\|\underline{u}_{\lambda}\right\|_{\mathbf{V}}^{2} .
\end{aligned}
$$


We will lower bound $\left\|\underline{u}_{\lambda}\right\|_{\mathbf{V}}^{2}$ in terms of $\|\lambda\|_{\mathbf{N}}^{2}$. Using the inf-sup condition for bilinear form $c$, there exists a constant $\gamma>0$ such that:

$$
\begin{aligned}
\gamma\|\lambda\|_{\mathbf{N}} & \leqslant \sup _{\underline{v} \in \mathbf{V}} \frac{c(\lambda, \underline{v})}{\|\underline{v}\|_{\mathbf{V}}}=\sup _{\underline{v} \in \mathbf{V}} \frac{-\left\langle A \underline{u}_{\lambda}+B^{*} p_{\lambda}, \underline{v}\right\rangle_{\mathbf{V}}}{\|\underline{v}\|_{\mathbf{V}}} \\
& \leqslant\left\|\underline{u}_{\lambda}\right\|_{\mathbf{V}}+\left\|p_{\lambda}\right\|_{\mathbf{M}}
\end{aligned}
$$

then, if we control $\left\|p_{\lambda}\right\|_{\mathbf{M}}$ in terms of $\left\|\underline{u}_{\lambda}\right\|_{\mathbf{V}}$ we obtain our bound. Next, as $\mathbf{M}$ is simply $L^{2}(\Omega)$, and for our bilinear form $b$ the inf-sup condition is achieved on functions with zero jumps, there exists a constant $\beta>0$ such that:

$$
\begin{aligned}
\beta\left\|p_{\lambda}\right\|_{0, \Omega} & \leqslant \sup _{v \in \mathbf{H}_{0}^{1}(\Omega), t \in \mathbb{R}} \frac{\left\langle B^{*} p_{\lambda}, \underline{v}\right\rangle}{\left(\|\nabla v\|_{0, \Omega}^{2}+t^{2}\right)^{1 / 2}} \\
& =\sup _{v \in \mathbf{H}_{0}^{1}(\Omega), t \in \mathbb{R}} \frac{-\left\langle A u_{\lambda}, \underline{v}\right\rangle}{\left(\|\nabla v\|_{0, \Omega}^{2}+t^{2}\right)^{1 / 2}} \leqslant\left\|\underline{u}_{\lambda}\right\|_{\mathbf{v} .}
\end{aligned}
$$

Then, $\gamma\|\lambda\|_{\mathbf{N}} \leqslant\left\|\underline{u}_{\lambda}\right\|_{\mathbf{V}}+\left\|p_{\lambda}\right\|_{\mathbf{M}} \leqslant\left(1+\beta^{-1}\right)\left\|\underline{u}_{\lambda}\right\|_{\mathbf{V}}$ and $\left\|\underline{u}_{\lambda}\right\|_{\mathbf{V}} \geqslant \gamma \beta(1+$ $\beta)^{-1}\|\lambda\|_{\mathbf{N}}$. Finally,

$$
(S \lambda, \lambda)_{\mathbf{N}}=\left\|\underline{u}_{\lambda}\right\|_{\mathbf{V}}^{2} \geqslant \frac{\gamma^{2} \beta^{2}}{(1+\beta)^{2}}\|\lambda\|_{\mathbf{N}}^{2}
$$

On the other hand, $S \lambda=-C u_{\lambda}$ implies $\|S \lambda\|_{\mathbf{N}} \leqslant\left\|\underline{u}_{\lambda}\right\|_{\mathbf{V}}$ and $\left\|\underline{u}_{\lambda}\right\|_{\mathbf{V}}^{2}=(S \lambda, \lambda)_{\mathbf{N}}$, then $\left\|\underline{u}_{\lambda}\right\|_{\mathbf{v}} \leqslant\|\lambda\|_{\mathbf{N}}$. As a conclusion

$$
\sigma^{2}\|\lambda\|_{\mathbf{N}}^{2} \leqslant(S \lambda, \lambda)_{\mathbf{N}} \leqslant\|\lambda\|_{\mathbf{N}}^{2}
$$

for $\sigma=\gamma \beta(1+\beta)^{-1}$.

\section{Finite dimensional approach: discrete dual and primal problems}

Now we introduce a discrete framework that will allow to obtain the dual problem in a finite dimensional setting and with the same properties. We pose problem (2.5) in discrete spaces $\mathbf{V}_{h}, \mathbf{M}_{h}$, and $\mathbf{N}_{h}$ and obtain inf-sup conditions for $b$ and $c$ uniformly with respect to the discretization parameter $h$. To further simplify we consider a conforming triangulation $\mathscr{T}_{h}$ of $\bar{\Omega}$ that contains the skeleton $\mathscr{E}_{0}$ as union of edges of triangles and so that on each edge only one partition is inherited from both sides. As usual, $h$ is the mesh size, i.e., $h=\max h_{\varkappa}$, where $\varkappa$ is a generic element of the mesh and $h_{\varkappa}$ is the longest side of $\varkappa$. As $\mathscr{T}_{h}$ is also compatible with the subdivision of $\Omega$, its restriction to each $\overline{\Omega_{s}}$ gives a mesh $\mathscr{T}_{h}^{s}$ on $\overline{\Omega^{s}}$. 
To fix ideas will use the Taylor-Hood finite element for the velocity and pressure pair on each subdomain. Define the family of subspaces $\left\{Y_{h}\right\}_{h} \subset$ $H_{0}^{1}(\Omega)$ and $\left\{Q_{h}\right\}_{h} \subset H^{1}(\Omega)$ given by

$$
\begin{aligned}
Y_{h} & =\left\{v \in H_{0}^{1}(\Omega): v_{\left.\right|_{\varkappa}} \in \mathbb{P}_{2}(\varkappa) \quad \forall \varkappa \in \mathscr{T}_{h}\right\} \\
Q_{h} & =\left\{p \in H^{1}(\Omega): p_{\left.\right|_{\varkappa}} \in \mathbb{P}_{1}(\varkappa) \quad \forall \varkappa \in \mathscr{T}_{h}\right\}
\end{aligned}
$$

where $\mathbb{P}_{r}(\varkappa)$ is the space of polynomials of degree less or equal to $r$ in the two variables $x$ and $y$. On each subdomain, we take also

$$
Y_{h}\left(\Omega^{s}\right)=Y_{h} \cap H^{1}\left(\Omega^{s}\right), \quad Q_{h}\left(\Omega^{s}\right)=Q_{h} \cap H^{1}\left(\Omega^{s}\right), \quad 1 \leqslant s \leqslant S .
$$

Consider now $\mathbf{X}_{h}=X_{h} \times X_{h}$, where $X_{h}$ is the broken version of $Y_{h}$ given by

$$
\begin{aligned}
X_{h}= & \left\{v \in L^{2}(\Omega): v^{s} \in Y_{h}\left(\Omega^{s}\right) \quad \forall s=1,2, \ldots, S\right. \\
& \text { and } v \text { is continuous at every cross point }\} \subset X
\end{aligned}
$$

define $\mathbf{V}_{h}=\mathbf{X}_{h} \times \mathbb{R}, \mathbf{M}_{h}=\prod_{s=1}^{S} Q_{h}\left(\Omega^{s}\right)$ and finally $\mathbf{N}_{h} \subset \mathbf{N}$ given by the restriction of functions in $\mathbf{X}_{h}$ to the skeleton $\mathscr{E}_{0}$. Then, we just place $h$ everywhere and compute $\underline{u_{h}}=\left(u_{h}, \tau\right) \in \mathbf{V}_{h}, p_{h}=\left\{p_{h}^{s}\right\}_{s} \in \mathbf{M}_{h}$ and $\lambda_{h}=\left\{\lambda_{h, e}\right\}_{e} \in \mathbf{N}_{h}$.

This problem can be written in terms of operators as we did before and proceed in the same manner to obtain the dual problem for the discrete Lagrange multipliers and with the same properties. The discrete uniform inf-sup condition for $c$ on the pair $\mathbf{V}_{h}$ and $\mathbf{N}_{h}$ is a well known result (see for instance Theorem 4 in [3]), that can be obtained using the standard finite element extension theorems or the extra regularity of solutions for elliptic problems in polygonal domains; it is also obtained on discrete functions with jumps.

Lemma 3.1. There exists a positive constant $\gamma>0$ independent of the mesh size such that for all $\mu_{h} \in \mathbf{N}_{h}$ :

$$
\sup _{\left(v_{h}, t\right) \in \mathbf{V}_{\mathbf{h}}} \frac{c\left(\mu_{h},\left(v_{h}, t\right)\right)}{\left\|\left(v_{h}, t\right)\right\|_{\mathbf{v}}} \geqslant \sup _{v_{h} \in \mathbf{X}_{h}} \frac{c\left(\mu_{h}, v_{h}\right)}{\left\|v_{h}\right\|_{\mathbf{X}}} \geqslant \gamma\left\|\mu_{h}\right\|_{\mathbf{N}} .
$$

Second, we check the inf-sup condition for $b$ and see that can be achieved with discrete continuous functions. This is more delicate and the idea is to use the stability of the pair $\mathbb{P}_{2}-\mathbb{P}_{1}$ locally on each subdomain $\Omega^{s}$ and that of the pair $\mathbb{P}_{2}-\mathbb{P}_{0}$ globally on $\Omega$.

Lemma 3.2. Assume that every triangle $\varkappa \in \mathscr{T}_{h}^{s}$ has at most one edge on $\partial \Omega^{s}$. Then, there exists a positive constant $\beta>0$ independent of the mesh size 
such that

$$
\sup _{\underline{v}_{h} \in \mathbf{V}_{h}} \frac{b\left(p_{h}, \underline{v}_{h}\right)}{\left\|\underline{v}_{h}\right\|_{\mathbf{v}}} \geqslant \beta\left\|p_{h}\right\|_{\mathbf{M}} \quad \forall p_{h} \in \mathbf{M}_{h}
$$

and the function $v_{h} \in \mathbf{V}_{h}$ that gives the maximum satisfies $v_{h} \in C^{0}(\bar{\Omega})$, i.e., its jumps across all interfaces are zero.

Proof. The pair $\left(Y_{h}\left(\Omega^{s}\right) \cap H_{0}^{1}\left(\Omega^{s}\right)\right)^{2}$ and $Q_{h}\left(\Omega^{s}\right) \cap L_{0}^{2}\left(\Omega^{s}\right)$ satisfies a uniform inf-sup condition on $\Omega^{s}$ because every triangle has at most one edge on $\partial \Omega^{s}$ (see Proposition 6.1 pp. 252 in the book by Brezzi-Fortin [6]). On the other hand, $\mathbf{X}_{h}$ contains the $\mathbb{P}_{2}$ continuous finite element functions which are enough to construct the Fortin operator (Lemma 1.1, page 117 in GiraultRaviart [11]), that allows to control pressures with vanishing average and that are constant on each subdomain. As a consequence (according also to Theorem 1.12 in Girault-Raviart [11]), there exists a function $v_{h}^{*} \in \mathbf{X}_{h} \cap C^{0}(\bar{\Omega})$ such that

$$
-\left(\operatorname{div}\left(v_{h}^{*}\right), p_{h}^{\prime}\right)_{\Omega}=\left\|p_{h}^{\prime}\right\|_{0, \Omega}^{2}, \quad\left\|\nabla v_{h}^{*}\right\|_{0, \Omega^{s}} \leqslant c_{2}\left\|p_{h}^{\prime}\right\|_{0, \Omega} \quad \forall p_{h}^{\prime} \in \mathbf{M}_{h} \cap L_{0}^{2}(\Omega) .
$$

Now, given $p_{h} \in \mathbf{M}_{h}$ take $p_{h}^{\prime}=p_{h}-p_{\Omega} \in \mathbf{M}_{h} \cap L_{0}^{2}(\Omega)$, where

$$
p_{\Omega}=|\Omega|^{-1} \int_{\Omega} p_{h} \mathrm{~d} x
$$

Then, using $\underline{v}_{h}^{*}=\left(v_{h}^{*}, t^{*}\right)$ with $t^{*}=-p_{\Omega}$, and that $v_{h}^{*}=0$ on $\partial \Omega$ the result follows as in Lemma 2.1.

\section{Dual and primal problems}

We cast the problem in terms of solving an equation for the discrete dual variable $\lambda_{h}$ set on the Lagrange multiplier space $\mathbf{N}_{h}$. Thanks to the discrete uniform inf-sup conditions, (2.7) is replicated in $\mathbf{N}_{h}$. As a consequence, we solve our dual problem in $\mathbf{N}_{h}$ via conjugate gradient method without preconditioner.

Conjugate Gradient Method (CG). For $\lambda_{0} \in \mathbf{N}_{h}$ compute the residual

$$
r_{0}=d_{0}=\ell-S \lambda_{0} \in \mathbf{N}_{h}
$$


and for $\delta_{m}=S d_{m}$ with $m \geqslant 0$ repeat

$$
\begin{aligned}
\alpha_{m} & =\frac{\left(r_{m}, r_{m}\right)_{\mathbf{N}}}{\left(d_{m}, \delta_{m}\right)_{\mathbf{N}}} \\
\lambda_{m+1} & =\lambda_{m}+\alpha_{m} d_{m} \\
r_{m+1} & =r_{m}-\alpha_{m} \delta_{m} \\
\beta_{m} & =\frac{\left(r_{m+1}, r_{m+1}\right)_{\mathbf{N}}}{\left(r_{m}, r_{m}\right)_{\mathbf{N}}} \\
d_{m+1} & =r_{m+1}+\beta_{m} d_{m}
\end{aligned}
$$

until the residual $r_{m}=\ell-S \lambda_{m}$ is small enough. Starting off with $\lambda_{0}=0$ the computation of the residual $r_{0, h}$ is given by $r_{0, h}=\ell_{h}=C \underline{u}_{0, h}$ where

$$
\underline{u}_{0, h}=A^{-1} F-A^{-1} B^{*}\left(B A^{-1} B^{*}\right)^{-1}\left(B A^{-1} F\right) \in \mathbf{V}_{h} .
$$

In terms of the primal problem, if we set $p_{0, h}=\left(B A^{-1} B^{*}\right)^{-1}\left(B A^{-1} F\right)$ this means solving $\left(\underline{u}_{0, h}, p_{0, h}\right) \in \mathbf{V}_{h} \times \mathbf{M}_{h}$ such that

$$
\begin{aligned}
\left\langle A \underline{u}_{0, h}, \underline{v}_{h}\right\rangle+\left\langle B^{*} p_{0, h}, \underline{v}_{h}\right\rangle & =\left\langle F, \underline{v}_{h}\right\rangle & & \forall \underline{v}_{h} \in \mathbf{V}_{h} \\
\left\langle B \underline{u}_{0, h}, q_{h}\right\rangle & =0 & & \forall q_{h} \in \mathbf{M}_{h} .
\end{aligned}
$$

Next, in the general step for the CG method, we set $d_{0, h}=r_{0, h}=C \underline{u}_{0, h}$ (drop some of the $h$ 's for simplicity), and for $m \geqslant 0$, once $d_{m}$ is obtained, we compute $\delta_{m}=S d_{m}=C \underline{w}_{m}$ where

$$
\underline{w}_{m}=A^{-1} C^{*} d_{m}-A^{-1} B^{*}\left(B A^{-1} B^{*}\right)^{-1}\left(B A^{-1} C^{*}\right) d_{m} \in \mathbf{V}_{h} .
$$

Again, in terms of the primal problem, if we set $q_{m}=\left(B A^{-1} B^{*}\right)^{-1}\left(B A^{-1} C^{*}\right) d_{m}$ then we solve $\left(\underline{w}_{m}, q_{m}\right) \in \mathbf{V}_{h} \times \mathbf{M}_{h}$ such that

$$
\begin{aligned}
\left\langle A \underline{w}_{m}, \underline{v}_{h}\right\rangle+\left\langle B^{*} q_{m}, \underline{v}_{h}\right\rangle & =\left\langle C^{*} d_{m}, \underline{v}_{h}\right\rangle & & \forall \underline{v}_{h} \in \mathbf{V}_{h} \\
\left\langle B \underline{w}_{m}, q_{h}\right\rangle & =0 & & \forall q_{h} \in \mathbf{M}_{h} .
\end{aligned}
$$

As a consequence, we have:

1. an external computational cycle, the CG for $S$ with a fixed number of iterations independent of the discretization parameter $h$ and

2. at each iteration of this external cycle, the resolution of a primal Stokeslike problem of the form: Find $\left(\underline{w}_{h}, q_{h}\right) \in \mathbf{V}_{h} \times \mathbf{M}_{h}$ such that

$$
\begin{aligned}
\left\langle A \underline{w}_{h}, \underline{v}_{h}\right\rangle+\left\langle B^{*} q_{h}, \underline{v}_{h}\right\rangle & =\left\langle\xi, \underline{v}_{h}\right\rangle & & \forall \underline{v}_{h} \in \mathbf{V}_{h} \\
\left\langle B \underline{w}_{h}, q_{h}\right\rangle & =0 & & \forall q_{h} \in \mathbf{M}_{h}
\end{aligned}
$$

where for the initial residual $r_{0}$ we have $\xi=F$ and for the iteration $m \geqslant 0$ we have $\xi=C^{*} d_{m}$. 


\subsection{Linear systems generated by the primal problem}

A closer look to the general form of this saddle point problem for the primal variables will show that the solution can be obtained mainly by means of independent block solves per subdomain. This is a similar computation process as in FETI-DP methods, but in this case each block will contain the standard stiffness matrix and the contributions from the boundary integrals. We order and split the unknowns $w_{h}=\left(w_{h}, \tau\right)$ and $q_{h}$ according to subdomains and cross points

$$
\left(x^{1}, x^{2}, \ldots, x^{S}, x^{C}, \tau\right)^{t} \text {. }
$$

Here $x^{s}=\left(\tilde{w}^{s}, q^{s}\right)^{t}$ are the dof's on each subdomain except those corresponding to the velocity field at the cross points and $x^{C}$ are the dof's at the cross points of the velocity field. As a consequence, in the linear system for the primal variables $M x=b$ the matrix $M$ has the following general block structure

$$
\left(\begin{array}{cccccccc}
M_{11} & M_{1,2} & \ldots & \ldots & \ldots & M_{1, S} & M_{1, C} & D_{1} \\
M_{21} & M_{2,2} & M_{2,3} & \ldots & \ldots & M_{2, S} & M_{2, C} & D_{2} \\
M_{31} & M_{3,2} & M_{3,3} & M_{3,4} & \ldots & M_{3, S} & M_{3, C} & D_{3} \\
\vdots & \ddots & \ddots & \ddots & \ddots & \vdots & \vdots & \vdots \\
\vdots & \ddots & \ddots & \ddots & \ddots & \vdots & \vdots & \vdots \\
M_{S, 1} & M_{S, 2} & \ldots & \ldots & M_{S, S-1} & M_{S, S} & M_{S, C} & D_{S} \\
M_{1, C}^{t} & M_{2, C}^{t} & \ldots & \ldots & M_{S-1, C}^{t} & M_{S, C}^{t} & M_{C, C} & 0 \\
D_{1}^{t} & D_{2}^{t} & \ldots & \ldots & D_{S-1}^{t} & D_{S}^{t} & 0^{t} & 1
\end{array}\right)\left(\begin{array}{c}
x^{1} \\
x^{2} \\
x^{3} \\
\vdots \\
\vdots \\
x^{S} \\
x^{C} \\
\tau
\end{array}\right)=\left(\begin{array}{c}
b^{1} \\
b^{2} \\
b^{3} \\
\vdots \\
\vdots \\
b^{S} \\
b^{C} \\
0
\end{array}\right)
$$

where the different blocks are of the form

$$
M_{s, s}=\left(\begin{array}{cc}
A_{s, s} & B_{s, s} \\
B_{s, s}^{t} & 0
\end{array}\right), \quad M_{s, s^{\prime}}=\left(\begin{array}{cc}
A_{s, s^{\prime}} & 0 \\
0 & 0
\end{array}\right), \quad M_{s, C}=\left(\begin{array}{c}
A_{s, C} \\
B_{s, C}^{t}
\end{array}\right), \quad M_{C, C}=A_{C, C}
$$

here each block $M_{s, s}$ is similar to a standard Stokes matrix on the subdomain $\Omega^{s}$, but with our interface contributions, each block $M_{s, s^{\prime}}\left(s \neq s^{\prime}\right)$ is sparse and contains the interaction through interfaces of the domain $\Omega^{s}$ with $\Omega^{s^{\prime}}$, the rectangular blocks $M_{s, C}$ contains the interaction with the crosspoints and $M_{C, C}$ contains the interaction of the crosspoints with themselves. Finally, the right hand sides are of the form

$$
b^{s}=\left(\left\{\left\langle\xi, \varphi_{j}^{s}\right\rangle\right\}_{j}, 0\right)^{t}, \quad b^{C}=\left(\left\{\left\langle\xi, \psi_{j}^{C}\right\rangle\right\}_{j}\right)^{t}
$$

(observe that $b^{C}=0$ when $\xi=C^{*} d_{m}$ ) and the vector blocks $D^{s}=\left(0,\left\{d_{j}^{s}\right\}_{j}\right)^{t}$ are formed by

$$
d_{j}^{s}=-\int_{\Omega} \varphi_{j}^{s}
$$


This linear system couples all the subdomains but can be solved by means of the Preconditioned Conjugate Gradient Method using as a preconditioner.

$$
P=\left(\begin{array}{ccccccc}
M_{11} & 0 & \ldots & \ldots & 0 & M_{1, C} & D_{1} \\
0 & M_{2,2} & 0 & \ldots & 0 & M_{2, C} & D_{2} \\
0 & 0 & M_{3,3} & 0 & \ddots & M_{3, C} & D_{3} \\
\vdots & \ddots & \ddots & \ddots & \ddots & \vdots & \vdots \\
\ldots & \ldots & \ldots & 0 & M_{S, S} & M_{S, C} & D_{S} \\
M_{1, C}^{t} & M_{2, C}^{t} & \ldots & M_{S-1, C}^{t} & M_{S, C}^{t} & M_{C, C} & 0 \\
D_{1}^{t} & D_{2}^{t} & \ldots & D_{S-1}^{t} & D_{S}^{t} & 0^{t} & 1
\end{array}\right) .
$$

As a consequence, $N=M-P$ is a sparse matrix that just contains the sparse blocks $M_{s, s^{\prime}}$ and does not need to be explicitly constructed. The main task here is the resolution of a linear system of the form $P x=b$ which is done via a Schur complement process in terms of basic equations for $x^{C}$ and $\tau$ as follows:

$$
\begin{gathered}
\left(M_{C, C}-\sum_{s=1}^{S} M_{s, C}^{t} M_{s, s}^{-1} M_{s, C}\right) x^{C}-\sum_{s=1}^{S} M_{s, C}^{t} M_{s, s}^{-1} D_{s} \tau=b^{C}-\sum_{s=1}^{S} M_{s, C}^{t} M_{s, s}^{-1} b^{s} \\
\sum_{s=1}^{S} D_{s}^{t} M_{s, S}^{-1} M_{s, C} x^{C}+\left(\sum_{s=1}^{S} D_{s}^{t} M_{s, S}^{-1} D_{s}-1\right) \tau=\sum_{s=1}^{S} D_{s}^{t} M_{s, s}^{-1} b^{s} .
\end{gathered}
$$

Equations (4.2)-(4.3) constitute the coarse problem for this method. The main task is performed with independent solves of the matrices $M_{s, s}$, i.e., computations of the form

$$
M_{s, s}^{-1} b^{s}, \quad M_{s, s}^{-1} M_{s, C}, \quad M_{s, s}^{-1} D_{s}
$$

and all of these can be performed independently. This Schur process is similar to that in FETI-DP methods.

\subsection{Condition number for the preconditioned conjugate gradient method}

When both $M$ and $P$ are symmetric positive definite and we solve $M x=b$ via a preconditioning $\left(P^{-1} M\right) x=P^{-1} b$ it is well known that

$$
k_{1}(P w, w) \leqslant(M w, w) \leqslant k_{2}(P w, w) \quad \forall w \in \mathbb{R}^{n}
$$

implies that the spectral condition number of $P^{-1} M$ is bounded by $k_{2} / k_{1}$, i.e.,

$$
\varkappa_{2}\left(P^{-1} M\right) \leqslant \frac{k_{2}}{k_{1}} .
$$


As a consequence, when the partition of $\Omega$ does not includes cross points, all jumps are controlled in terms of the gradients and the couplings in $M$ can be removed safely. Then, $k_{1}$ and $k_{2}$ are independent of $h$ and we recover a condition number for the primal problem independent of $h$.

On the other hand, when cross points are present in the partition, we need to use the classical result by Mandel and Brezina [15] that allows to bound the internal jumps in terms of the gradient norm but using a $\log (H / h)$ dependent norm. In this case we recover the standard condition number for the primal problem of the form

$$
\varkappa_{2}\left(P^{-1} M\right) \leqslant C(1+\log (H / h))^{2} .
$$

\section{Some numerical examples}

All the numerical tests that we present have been performed with freefem++ [10]. With the available tools we are able to compute the mass matrices for the $H^{1 / 2}$ and $H_{00}^{1 / 2}$ scalar products for any edge and place them in the correct entries of the global stiffness matrix for any subdomain. Any traces of any finite element function can be used and, when it comes to compute these unusual scalar products on an edge, the same code handles conforming and nonconforming triangulations by simply assigning different discretization points on each side of the edge. The method has not been implemented in parallel just for simplicity, although freefem++ can also do it.

\subsection{Conforming triangulations}

For $L=1,2, \ldots$ integer we consider a test defined on $\Omega=(0, L) \times(0,1)$ with exact solution

$$
u(x, y)=\left(\begin{array}{c}
-\sin ^{3}\left(\pi x L^{-1}\right) \sin ^{2}(\pi y) \cos (\pi y) \\
-L^{-1} \sin ^{2}\left(\pi x L^{-1}\right) \sin ^{3}(\pi y) \cos \left(\pi x L^{-1}\right)
\end{array}\right), \quad p(x, y)=\frac{x^{2}}{L^{2}}-y^{2}
$$

and consider a partition of $\Omega$ into $L$ subdomains given by $\Omega^{s}=(s-1, s) \times(0,1)$ for $s=1,2, \ldots, L$. For the dual problem we always start with $\lambda_{0, e}=0$ on each interface $\Gamma_{e}$. In this example there is no need to add the jumps in the elliptic parts because there are no cross points. As a consequence, there is no need for a PCG in the internal cycle because the blocks $M_{s, t}$ are null for $s \neq t$. Table 1 shows that the iteration count for the dual problem is mesh independent on different configurations

Table 2 shows the relative errors with respect to the true solution $u$ and $p$ compared with $u_{h}^{\mathrm{DDM}}$ and $p_{h}^{\mathrm{DDM}}$, the approximation obtained on $\Omega$ using our method. 
Table 1.

Mesh independent iteration counts in the dual problem for first test.

\begin{tabular}{rccc}
\hline$L$ & $h=1 / 24$ & $h=1 / 48$ & $h=1 / 96$ \\
\hline 4 & 17 & 17 & 17 \\
8 & 23 & 24 & 24 \\
16 & 37 & 39 & 39 \\
\hline
\end{tabular}

Table 2.

Relative errors in velocity field and pressure for first test.

\begin{tabular}{lccccccc}
\hline$e u(h)$ & $h=1 / 24$ & $h=1 / 48$ & $h=1 / 96$ & $e p(h)$ & $h=1 / 24$ & $h=1 / 48$ & $h=1 / 96$ \\
\hline$L=4$ & $2.1 \mathrm{e}-04$ & $2.6 \mathrm{e}-05$ & $3.5 \mathrm{e}-6$ & $L=4$ & $6.7 \mathrm{e}-04$ & $1.6 \mathrm{e}-04$ & $4.0 \mathrm{e}-5$ \\
$L=8$ & $1.8 \mathrm{e}-04$ & $2.3 \mathrm{e}-05$ & $3.0 \mathrm{e}-6$ & $L=8$ & $6.8 \mathrm{e}-04$ & $1.6 \mathrm{e}-04$ & $4.2 \mathrm{e}-5$ \\
$L=16$ & $1.7 \mathrm{e}-04$ & $2.2 \mathrm{e}-05$ & $2.9 \mathrm{e}-6$ & $L=16$ & $6.8 \mathrm{e}-04$ & $1.7 \mathrm{e}-04$ & $4.3 \mathrm{e}-5$ \\
\hline
\end{tabular}

As a second test, we take on $\Omega=(0,1)^{2}$ the exact solution given by the same velocity field $u(x, y)$ as before with $L=1$, pressure given by

$$
p(x, y)=(x-0.25)^{2}(y-0.25)^{2}
$$

and partition $\Omega$ into four equal subdomains with a cross point at $(0.5,0.5)$. Table 3 shows the results and how the number of iterations is also independent of the mesh size.

\subsection{Nonconforming triangulations}

We first consider $\Omega=(0,2) \times(0,1)$ and split it into two subdomains through a curved interface and then consider $\Omega=(0,2) \times(0,2)$ with a floating subdomain, the disk centered at the point $(0.75,1)$ with radius $r=0.5$. In this last

Table 3.

A cross point test. Number of iterations for dual problem, primal problems and relative errors on velocity and pressure.

\begin{tabular}{cccccc}
\hline$h$ & $\begin{array}{c}\text { Dual } \\
\text { \# Iters }\end{array}$ & $\begin{array}{c}\text { initial PCG } \\
\text { \# Iters }\end{array}$ & $\begin{array}{c}\text { final PCG } \\
\text { \# Iters }\end{array}$ & $e u(h)$ & $e p(h)$ \\
\hline $1 / 12$ & 7 & 22 & 20 & $6.9 \mathrm{e}-4$ & $4.2 \mathrm{e}-3$ \\
$1 / 24$ & 7 & 21 & 20 & $8.8 \mathrm{e}-5$ & $1.0 \mathrm{e}-3$ \\
$1 / 48$ & 7 & 23 & 21 & $1.2 \mathrm{e}-5$ & $2.5 \mathrm{e}-4$ \\
$1 / 96$ & 7 & 23 & 23 & $1.4 \mathrm{e}-6$ & $8.3 \mathrm{e}-5$ \\
\hline
\end{tabular}




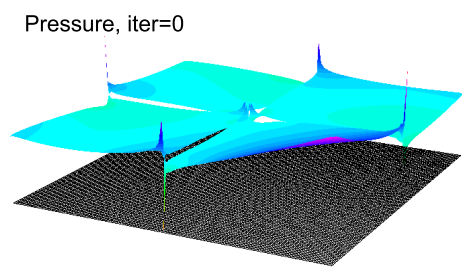

Pressure, iter $=2$

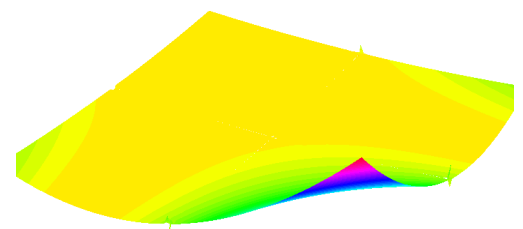

Pressure, iter $=1$

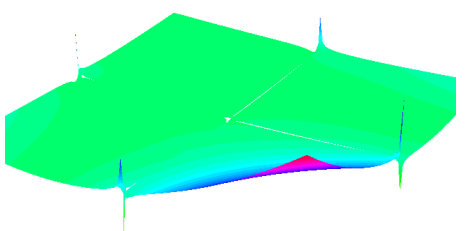

Pressure, iter $=6$

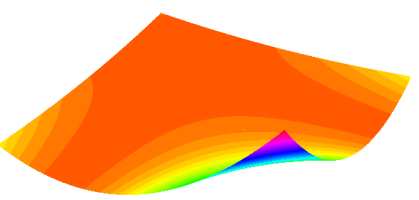

Figure 2. Evolution of the pressure field in the case of four subdomains with a cross point $(h=1 / 48)$.

configuration there are no cross points and we just work with the $H^{1 / 2}$ scalar product on the disk boundary. We compute the solution for the right hand side given by

$$
f_{1}(x, y)=(x-0.4)^{2}(y-0.8)^{3}, \quad f_{2}(x, y)=-(x-0.4)^{2}(y-0.5)^{3} .
$$

We use first a conforming mesh and next a nonconforming mesh for the discretization and approximate the disk border accordingly. Figure 3 and 4 show the different triangulations of $\Omega$ used while the results are in Figs 5-7.

\section{Conclusions}

The purpose of this paper has been to present an abstract setting for a variation of FETI-DP mortar method for Incompressible Stokes problem that has a matrix iteration for the dual problem symmetric positive definite with a mesh independent condition number. When solving this dual problem with Conjugate Gradient we face a primal problem that it is solved with the preconditioning conjugate gradient and using independent block computations. When the partition has cross points, this preconditioning brings back the well known slight dependency on $\log (H / h)$ for the condition number in the standard FETI-DP mortar methods. 


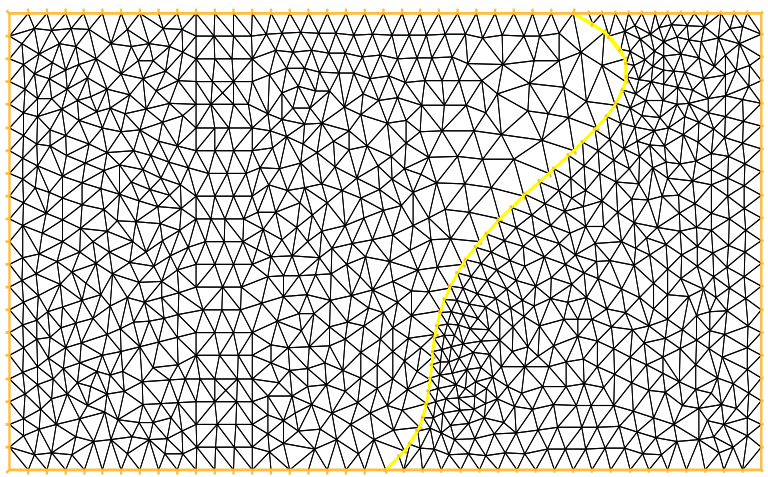

Figure 3. Triangulation for the two subdomain test with nonconforming meshes and curved interface.
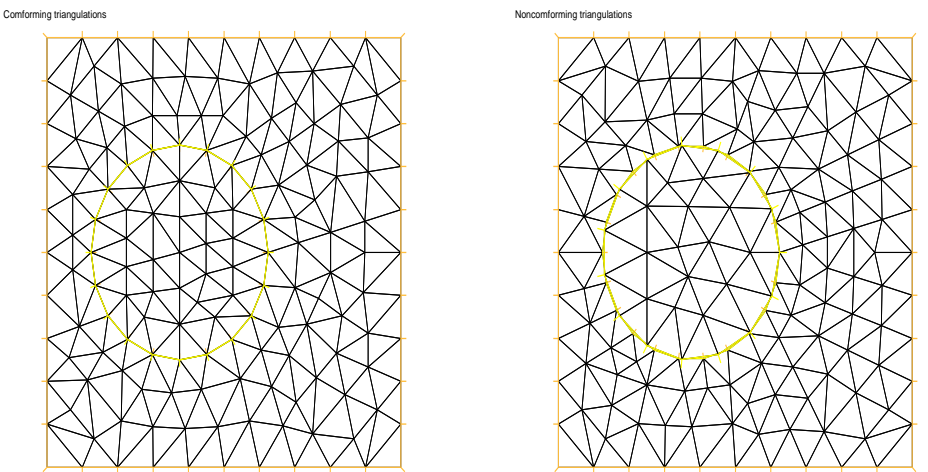

Figure 4. Triangulations for the floating subdomain test: from left to right, conforming mesh with 20 grid points to discretize the disk boundary and nonconforming triangulations with 22 and 15 grid points to discretize the disk boundary from outside and inside respectively. 


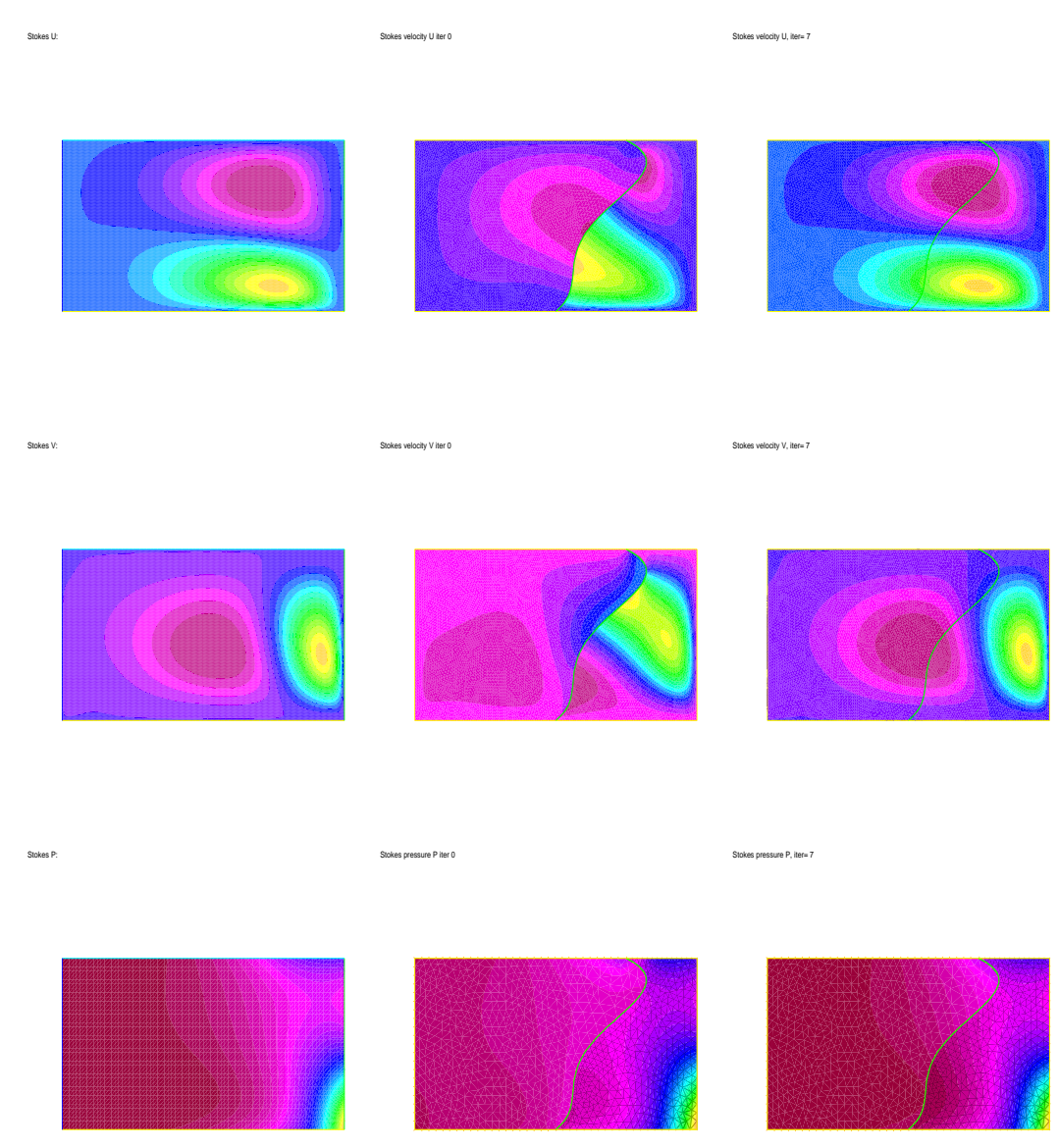

Figure 5. Two subdomain test with nonconforming meshes and curved interface; from left to right: fine mesh computation, first iteration and last iteration for velocities $U, V$, and pressure $P$. 

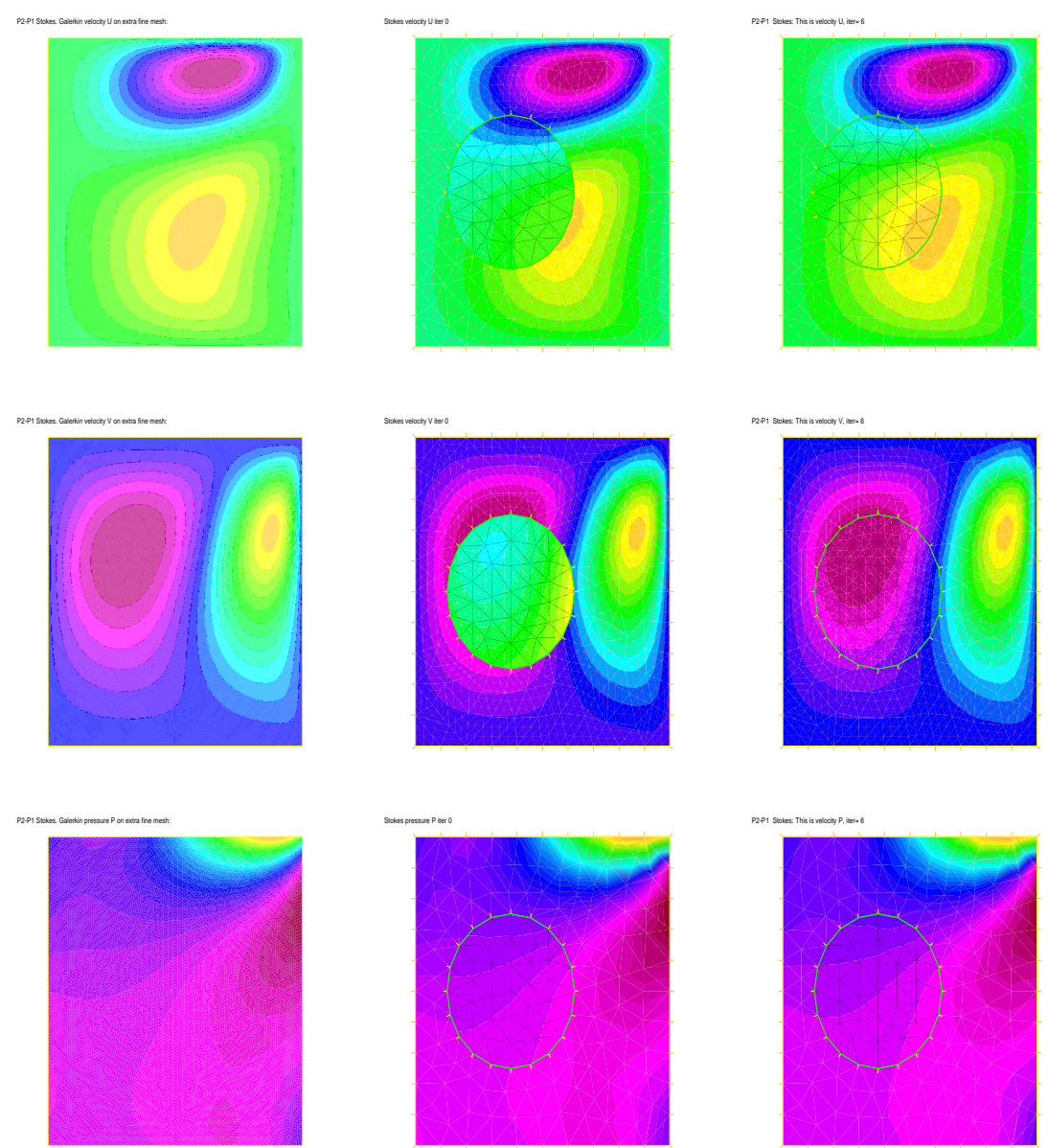

Figure 6. Floating subdomain test with conforming meshes; from left to right: fine mesh computation, first iteration and last iteration for velocities $U, V$, and pressure $P$. 

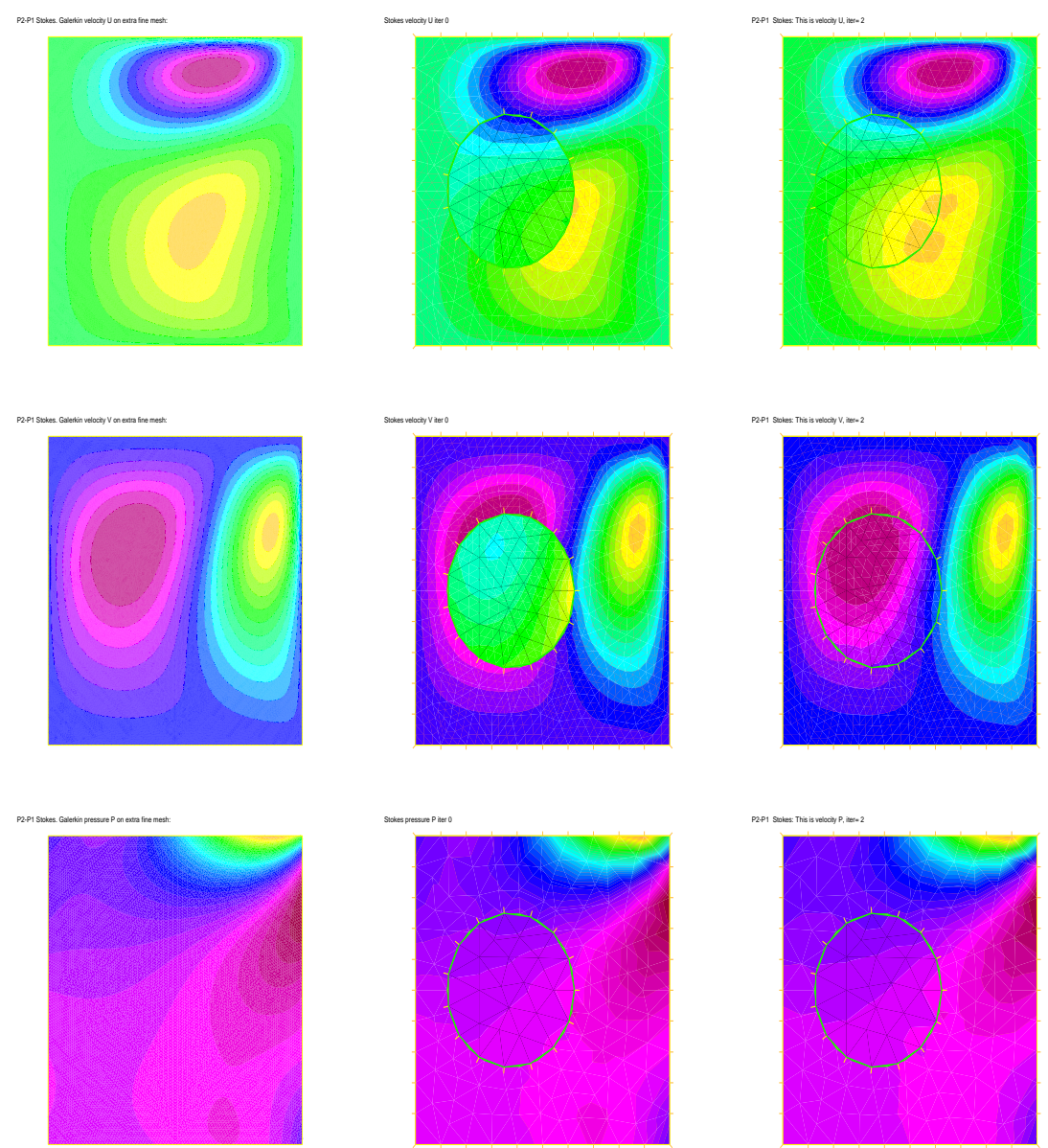

Figure 7. Floating subdomain test with non conforming meshes; from left to right: fine mesh computation, first iteration and last iteration for velocities $U, V$, and pressure $P$. 
The advantage of the continuous framework introduced is the clear sight of the effect of condensing all information on subdomains and interfaces before the discrete work starts and the use of the most appropriated norms on subdomains and interfaces that make no necessary the use of mesh dependent norms for obtaining stability. The clear disadvantage is the need for computing these $H^{1 / 2}$ norms, although we have shown it is feasible.

Many other alternatives are present in the literature, see the works by Li [14], Kim et al. [13], Achdou et al. [1], etc., and, as far as we know, our approach is original. In future work we would like to compare our method with standard mortar and FETI-DP methods and extract conclusions. Another interesting research could be the design of numerical tests with moderate to large coarse problems and, therefore, study the large scale computational properties (i.e. numerical scalability) of our approach.

Acknowledgments. The authors want to thank Professor Frédéric Hecht and his team for the development of the freefem++ project. We also would like to thank Professors Frédéric Nataf and Tomás Chacón Rebollo for many valuable comments on this research. Finally, we want to thank the financial support from Junta de Andalucia, Spain, via the research project P07-FQM-02538 'Freefem3d: Aplicaciones a la simulación numérica de flujos medioambientales en el entorno andaluz'.

\section{References}

1. Y. Achdou, Yu. A. Kuznetsov, and O. Pironneau, Substructuring preconditioners for the $Q_{1}$ mortar element method. Numer. Math. (1995) 71, No. 4, 419-449.

2. F. Ben Belgacem, The Mortar finite element method with Lagrange multipliers. $\mathbf{N u}$ merische Math. (1999) 84, 173-197.

3. C. Bernardi, T. Chacón Rebollo, and E. Chacón Vera, A FETI method with a mesh independent condition number for the iteration matrix. Comp. Meth. Appl. Mech. Engrg. (2008) 197, No. 13-16, $1410-1429$.

4. C. Bernardi, Y. Maday, and A.T. Patera, A new nonconforming approach to domain decomposition: the mortar element method. In: Collège de France Seminar XI (Eds. H. Brezis and J.-L. Lions). Pitman, 1994, pp. 13-51.

5. D. Braess, W. Dahmen, and C. Wieners, A multigrid algorithm for the mortar finite element method. SIAM J. Numer. Anal. (1999) 37, $48-69$.

6. F. Brezzi and M. Fortin, Mixed and Hybrid Finite Element methods. Springer-Verlag, Berlin, 1991.

7. E. Chacón Vera, A continuous framework for FETI-DP with a mesh independent condition number for the dual problem. Comp. Meth. Appl. Mech. Engrg. (2009) 198, 2470-2483.

8. M. Dryja and O.B. Widlund, A FETI-DP method for a mortar discretization of elliptic 
problems. In: Recent Developments in Domain Decomposition Methods (Zurich, 2001), Lect. Notes in Comput. Sci. Engrg., Vol.23, Springer, Berlin, 2002, pp. 41-52.

9. C. Farhat, M. Lesoinne, P. LeTallec, K. Pierson, and D. Rixen, FETI-DP: a dual-primal unified FETI method, Part I: A faster alternative to the two level FETI method. Int. J. Numer. Meth. Engrg. (2001) 50, $1523-1544$.

10. http://www.freefem.org/ff++

11. V. Girault and P.-A. Raviart, Finite Element Methods for Navier-Stokes Equations. Theory and Algorithms. Springer Series in Computational Mathematics, Vol. 5. Springer-Verlag, Berlin, 1986.

12. P. Grisvard, Singularities in Boundary Value Problems. Recherches en Mathématiques Appliquées 22, Masson, 1992.

13. H. H. Kim and C.-O. Lee, A FETI-DP formulation for two dimensional Stokes problem on nonmatching grids. Math. Comp. (1996) 65, No. 216, 1387-1401.

14. J. Li, A dual-primal FETI method for incompressible Stokes equations. Tech. Report 816. Department of Computer Science, Courant Institute, New York University, 2001.

15. J.Mandel and M. Brezina, Balancing domain decomposition for problems with large jumps in coefficients. Math. Comp. (1996) 65, No. 216, 1387-1401.

16. P. A. Raviart and J. M. Thomas, Primal hybrid finite element methods for second order elliptic equations Math. Comp. (1977) 31, No. 138, 391-413. 